\title{
Erratum to: The methyl donor S-adenosylmethionine potentiates doxorubicin effects on apoptosis of hormone-dependent breast cancer cell lines
}

\author{
Concetta Paola Ilisso $^{1}$ - Maria Castellano ${ }^{1}$ - Silvia Zappavigna ${ }^{1} \cdot$ Angela Lombardi $^{1}$. \\ Giovanni Vitale $^{2,3}$ - Alessandra Dicitore ${ }^{3}$ - Giovanna Cacciapuoti ${ }^{1}$. Michele Caraglia ${ }^{1}$. \\ Marina Porcelli ${ }^{1}$
}

Published online: 8 July 2016

(C) Springer Science+Business Media New York 2016

Erratum to: Endocrine (2015) 50:212-222 DOI: $10.1007 / \mathrm{s} 12020-014-0484-7$

Unfortunately, the original publication of the article contained an error in the affiliation section. The correct affiliation for authors A. Dicitore and G. Vitale should be Laboratory of Endocrine and Metabolic Research, Istituto Auxologico Italiano IRCCS, Via Zucchi 18, Cusano Milanino (MI), 20095 Milan, Italy.

This has been corrected with this erratum.

The online version of the original article can be found under doi: 10.1007/s12020-014-0484-7.

Silvia Zappavigna

silvia.zappavigna@unina2.it

1 Department of Biochemistry, Biophysics and General Pathology, Second University of Naples, Via L. De Crecchio 7, Naples 80138, Italy

2 Department of Clinical Sciences and Community Health, University of Milan, Milan, Italy

3 Laboratory of Endocrine and Metabolic Research, Istituto Auxologico Italiano IRCCS, Via Zucchi 18, Cusano Milanino (MI), 20095, Milan, Italy 